\title{
Bayesian inference in finite population sampling under measurement error model ${ }^{\dagger}$
}

\author{
You Mee Goo ${ }^{1}$. Dal Ho Kim² \\ ${ }^{12}$ Department of Statistics, Kyungpook National University \\ Received 3 October 2012, revised 7 November 2012, accepted 12 November 2012
}

\begin{abstract}
The paper considers empirical Bayes (EB) and hierarchical Bayes (HB) predictors of the finite population mean under a linear regression model with measurement errors. We discuss how to calculate the mean squared prediction errors of the EB predictors using jackknife methods and the posterior standard deviations of the HB predictors based on the Markov Chain Monte Carlo methods. A simulation study is provided to illustrate the results of the preceding sections and compare the performances of the proposed procedures.
\end{abstract}

Keywords: Empirical Bayes, finite population mean, Gibbs sampler, hierachical Bayes, jackknife method, mean squared prediction error, posterior standard deviation.

\section{Introduction}

We consider a finite population $\mathcal{U}$ with units labeled $1,2, \ldots, N$. Let $y_{i}$ denote the value of a single characteristic attached to the unit $i$. The vector $\boldsymbol{y}=\left(y_{1}, \ldots, y_{N}\right)^{T}$ is the unknown state of nature, and is assumed to belong to $\Theta=R^{N}$. Here we concern exclusively about the finite population mean $\gamma=N^{-1} \sum_{i=1}^{N} y_{i}$. A subset $s$ of $\{1,2, \ldots, N\}$ is called a sample. A sample $s$ of size $n$ is selected from $\mathcal{U}$ according to some specified sampling plan. And let $\bar{s}=\mathcal{U}-\boldsymbol{s}$ be the unobserved part of $\mathcal{U}$.

In this paper we use the superpopulation approach (Cassel et al., 1977) to survey sampling. Under this perspective, according to the conditionality principle (Basu, 1975), the sampling plan is not relevant for inference. Extensive bibliographies on the model-based superpopulation approach are given in Bolfarine and Zacks (1992). Throughout, we will use the notations $\boldsymbol{y}=\left(y_{1}, \ldots, y_{n}, y_{n+1}, \ldots, y_{N}\right)^{T}$ and $\boldsymbol{y}=\left(\boldsymbol{y}_{s}^{T}, \boldsymbol{y}_{\bar{s}}^{T}\right)^{T}$ with $\boldsymbol{y}_{s}=\left(y_{1}, \ldots, y_{n}\right)^{T}, \boldsymbol{y}_{\bar{s}}=$ $\left(y_{n+1}, \ldots, y_{N}\right)^{T}$. Also write $\mathbf{1}_{N-n}$ as the $N-n$ dimensional column vector of 1 's, $\boldsymbol{J}_{N-n}=$ $\mathbf{1}_{N-n} \mathbf{1}_{N-n}^{T}$ and $\boldsymbol{I}_{N-n}$ as the identity matrix of order $N-n$.

Measurement errors may occur when the measuring device is biased or inaccurate. Regarding human populations, the respondents may not possess accurate information or they may give biased information. As shown in Table 1.1 of Fuller (1987), simple characteristics like sex

$\dagger$ This Research was supported by Kyungpook National University Research Fund, 2010.

1 Ph.D. candidate, Department of Statistics, Kyungpook National University, Daegu 702-701, Korea.

2 Corresponding author: Professor, Department of Statistics, Kyungpook National University, Daegu 702-701, Korea. E-mail: dalkim@knu.ac.kr 
or age may also present some measurement errors. More complex population characteristics like unemployment, income or salary may present a much more serious measurement bias. According to Fuller (1987), measurement error is about 15 percent of the total variation for income. The effect of such kind of errors upon estimated regression coefficients has long been recognized as a serious problem. Cochran (1968) and Fuller $(1975,1987)$ are references reporting distortions that are introduced into the regression coefficient estimates when the variables in the regression equation are measured with errors. There has been little explicit analytical treatment of prediction problem for the finite population mean under regression superpopulation models with measurement errors.

We consider the superpopulation model

$$
\begin{aligned}
& y_{i}=\alpha+\beta x_{i}+e_{i}, i=1, \ldots, N \\
& X_{i}=x_{i}+\eta_{i}, i=1, \ldots, N
\end{aligned}
$$

It is assumed that the $x_{i}, \eta_{i}$ and $e_{i}$ are mutually independent with $x_{i} \stackrel{i i d}{\sim} N\left(\mu_{x}, \sigma_{x}^{2}\right), \eta_{i} \stackrel{i i d}{\sim}$ $N\left(0, \sigma_{\eta}^{2}\right)$ and $e_{i} \stackrel{i i d}{\sim} N\left(0, \sigma_{e}^{2}\right)$. The vector of model parameters is denoted by $\phi=\left(\alpha, \beta, \mu_{x}, \sigma_{x}^{2}\right.$, $\left.\sigma_{\eta}^{2}, \sigma_{e}^{2}\right)^{T}$. Also we assume that $\mathbf{X}=\left(X_{1}, X_{2}, \ldots, X_{N}\right)^{T}$ is known. Similarly to $\boldsymbol{y}$, we use the notations $\boldsymbol{X}=\left(\boldsymbol{X}_{s}^{T}, \boldsymbol{X}_{\bar{s}}^{T}\right)^{T}$ with $\boldsymbol{X}_{s}=\left(X_{1}, \ldots, X_{n}\right)^{T}, \boldsymbol{X}_{\bar{s}}=\left(X_{n+1}, \ldots, X_{N}\right)^{T}$.

Bolfarine and Cordani (1993) provided the likelihood-based inferences on the slope parameter of a simple linear regression model with measurement errors when the reliability ratio is known. Bolfarine et al. (1996) considered the frequentist approach to the prediction of the finite population total under regression superpopulation model with measurement errors. They study the asymptotic behavior of the naive predictor based on the ordinary least squares estimator as well as a bias-adjusted estimator, establishing the asymptotic normality. Later Torabi et al. (2009) justified that the naive predictor given in Bolfarine et al. (1996) is essentially identical to the EB predictor.

In this article, we consider $\mathrm{EB}$ and $\mathrm{HB}$ predictors of finite population mean when the covariate, say $x$, is measured with error. We also assume that $x$ is stochastic. Here the EB procedure need to estimate the hyperparameters, and does not require any approximation of the posterior. The HB procedure also does not rely any normal approximation.

The outline of the remaining section is as follows. In Section 2, we provide the EB and HB predictors of finite population mean where the covariates are measured with error. Also we discuss how to calculate the mean squared prediction error (MSPE) of the EB predictors using jackknife methods and the posterior standard deviation of the HB predictors based on the Markov Chain Monte Carlo (MCMC) methods. In Section 3, a simulation study is provided to illustrate the results of the preceding sections and compare the performances of the proposed procedures. Finally we provide the summary and conclusion.

\section{EB and HB predictors of the finite population mean}

A sample $s$ of size $n$ is drawn from the finite population and the sample data is denoted by $\left(y_{i}, X_{i} ; i \in s\right)$. From (1.1) and (1.2), the incidental parameters $x_{i}$ can be eliminated in such a way that $\left(y_{i}, X_{i}\right)$ has a bivariate normal distribution with

$$
\left(\begin{array}{c}
y_{i} \\
X_{i}
\end{array}\right) \sim N\left[\left(\begin{array}{c}
\alpha+\beta \mu_{x} \\
\mu_{x}
\end{array}\right),\left(\begin{array}{cc}
\beta^{2} \sigma_{x}^{2}+\sigma_{e}^{2} & \beta \sigma_{x}^{2} \\
\beta \sigma_{x}^{2} & \sigma_{x}^{2}+\sigma_{\eta}^{2}
\end{array}\right)\right], i=1, \ldots, N
$$


Using the well-known properties of the bivariate normal distribution, it follows that

$$
y_{i} \mid X_{i} \stackrel{i n d}{\sim} N\left[\mu_{y}+\beta k_{x}\left(X_{i}-\mu_{x}\right), \sigma_{e}^{2}+\beta^{2} \sigma_{x}^{2}\left(1-k_{x}\right)\right], i=1, \ldots, N
$$

where $\mu_{y}=\alpha+\beta \mu_{x}$ and $k_{x}=\sigma_{x}^{2} /\left(\sigma_{x}^{2}+\sigma_{\eta}^{2}\right)$. We are interested in the estimation of finite population mean $\gamma=N^{-1} \sum_{i=1}^{N} y_{i}$ from the sample data. It can be rewritten by $\gamma=$ $N^{-1}\left(\mathbf{1}_{n}^{T} \boldsymbol{y}_{s}+\mathbf{1}_{N-n}^{T} \boldsymbol{y}_{\bar{s}}\right)$.

First we derive the EB predictor of $\gamma$. The Bayes predictor of $\gamma$ under squared error loss is

$$
\hat{\gamma}^{B}=E\left(\gamma \mid \boldsymbol{y}_{s}, \boldsymbol{X}, \boldsymbol{\phi}\right)=(1-f) \bar{y}_{s}+N^{-1} \mathbf{1}_{N-n}^{T} E\left(\boldsymbol{y}_{\bar{s}} \mid \boldsymbol{y}_{s}\right),
$$

where $f=(N-n) / N$ is the finite population correction factor and $\bar{y}_{s}=n^{-1} \sum_{i=1}^{n} y_{i}$. The basic problem in finite population sampling is to draw predictive inference about $\boldsymbol{y}_{\bar{s}}$ conditional on $\boldsymbol{y}_{s}$. Since the conditional distribution of $\boldsymbol{y}_{\bar{s}}$ given $\boldsymbol{y}_{s}$ is given by

$$
\boldsymbol{y}_{\bar{s}} \mid \boldsymbol{y}_{s} \sim N\left[\mu_{y} \mathbf{1}_{N-n}+\beta k_{x}\left(\boldsymbol{X}_{\bar{s}}-\mu_{x} \mathbf{1}_{N-n}\right),\left\{\sigma_{e}^{2}+\beta^{2} \sigma_{x}^{2}\left(1-k_{x}\right)\right\} \boldsymbol{I}_{N-n}\right],
$$

we have $E\left(\boldsymbol{y}_{\bar{s}} \mid \boldsymbol{y}_{s}\right)=\mu_{y} \mathbf{1}_{N-n}+\beta k_{x}\left(\boldsymbol{X}_{\bar{s}}-\mu_{x} \mathbf{1}_{N-n}\right)$ for all $i \in \bar{s}$. Thus the Bayes predictor of $\gamma$ is given by

$$
\hat{\gamma}^{B}=(1-f) \bar{y}_{s}+f \mu_{y}+f \beta k_{x}\left(\bar{X}_{\bar{s}}-\mu_{x}\right) .
$$

Also, the posterior variance of $\gamma$ given $\phi$ is

$$
V\left(\gamma \mid \boldsymbol{y}_{s}, \boldsymbol{X}, \boldsymbol{\phi}\right)=\frac{1}{N} f\left\{\sigma_{e}^{2}+\beta^{2} \sigma_{x}^{2}\left(1-k_{x}\right)\right\} .
$$

Note that $V\left(\gamma \mid \boldsymbol{y}_{s}, \boldsymbol{X}, \boldsymbol{\phi}\right)$ does not depend on $\boldsymbol{y}_{s}$ and $\boldsymbol{X}$. Hence the MSPE of $\hat{\gamma}^{B}, E\left(\hat{\gamma}^{B}-\gamma\right)^{2}$, is equal to the posterior variance of $\gamma$. Also, note that the posterior variance of $\gamma$ depends only on $\boldsymbol{\delta}=\left(\beta, \sigma_{x}^{2}, \sigma_{\eta}^{2}, \sigma_{e}^{2}\right)^{T}$. We denote $g_{1}(\boldsymbol{\delta}) \equiv M S P E\left(\hat{\gamma}^{B}\right)=E\left(\hat{\gamma}^{B}-\gamma\right)^{2}$. If $N$ is large and $n / N \approx 0$, then $g_{1}(\boldsymbol{\delta}) \approx 0$.

The EB predictor $\hat{\gamma}^{E B}$ of $\gamma$ is obtained by replacing $\phi$ in the Bayes predictor $\hat{\gamma}^{B}$ by a consistent estimator $\hat{\phi}$. The components of $\phi$ are unknown and need to be estimated from the data. Let $\bar{X}_{s}=n^{-1} \sum_{i=1}^{n} X_{i}, S S_{X}=\sum_{i=1}^{n}\left(X_{i}-\bar{X}_{s}\right)^{2}, S S_{y}=\sum_{i=1}^{n}\left(y_{i}-\bar{y}_{s}\right)^{2}$, $S_{y X}=\sum_{i=1}^{n}\left(X_{i}-\bar{X}_{s}\right)\left(y_{i}-\bar{y}_{s}\right), M S_{X}=(n-1)^{-1} S S_{X}, M S_{y}=(n-1)^{-1} S S_{y}$ and $M S_{y X}=$ $(n-1)^{-1} S_{y X}$.

Under some regularity conditions, $\bar{y}_{s}$ and $\bar{X}_{s}$ are consistent estimator of $\mu_{y}$ and $\mu_{x}$, respectively, i.e., $\hat{\mu}_{y}=\bar{y}_{s}, \hat{\mu}_{x}=\bar{X}_{s}$. Under the superpopulation model (1.1) and (1.2), it can be shown that (see Fuller, 1987) $E\left[\hat{\beta}_{O L S}\right]=E\left(\frac{S_{Y X}}{S S_{X}}\right)=k_{x} \beta$, where $\hat{\beta}_{O L S}$ is the ordinary least-squares estimator of $\beta$, and thus $k_{x} \beta$ is consistently estimated by $\hat{\beta}_{O L S}$. Thus the EB predictor of $\gamma$ is given by

$$
\hat{\gamma}^{E B}=\bar{y}_{s}+f\left(\bar{X}_{\bar{s}}-\bar{X}_{s}\right) \hat{\beta}_{O L S} .
$$

Now we obtain a nearly unbiased estimator of $\operatorname{MSPE}\left(\hat{\gamma}^{E B}\right)=E\left(\hat{\gamma}^{E B}-\gamma\right)^{2}$, using the jackknife methods proposed by Jiang et al. (2002) and Chen and Lahiri (2002). We have the following orthogonal decomposition:

$$
M S P E\left(\hat{\gamma}^{E B}\right)=E\left(\hat{\gamma}^{B}-\gamma\right)^{2}+E\left(\hat{\gamma}^{E B}-\hat{\gamma}^{B}\right)^{2}=M_{1}+M_{2}
$$


where $M_{1}=g_{1}(\boldsymbol{\delta})$ is given by $(2.2)$.

A plug-in estimator of $g_{1}(\boldsymbol{\delta})$ is $g_{1}(\hat{\boldsymbol{\delta}})$. Following Fuller (1987), when we assume $\sigma_{\eta}^{2}$ to be known, the estimators are given by $\hat{\sigma}_{x}^{2}=M S_{X}-\sigma_{\eta}^{2}$ and $\hat{\sigma}_{e}^{2}=M S_{y}-\hat{\beta} M S_{y X}$ where $\hat{\beta}=S S_{y X} /\left\{S S_{X}-\sigma_{\eta}^{2}\right\}$. We apply the jackknife method of bias reduction to $g_{1}(\hat{\boldsymbol{\delta}})$ to get a nearly unbiased estimator of $M_{1}=g_{1}(\boldsymbol{\delta})$. Let $\hat{\boldsymbol{\phi}}_{-l}$ be the estimator of $\boldsymbol{\phi}$ obtained by deleting the $l$ th data set $\left(\boldsymbol{y}_{s}^{(l)}, \boldsymbol{X}_{s}^{(l)}\right)$ from the full data set $\left(y_{i}, X_{i} ; i \in s\right)$ and then applying the method-of-moments. This calculation is done for each $l$ in turn to get $n$ estimators of $\phi$ $:\left(\hat{\phi}_{-l} ; l=1, \ldots, n\right)$. A jackknife estimator of $M_{1}$ is given by

$$
\hat{M}_{1 J}=g_{1}(\hat{\boldsymbol{\delta}})-\sum_{l=1}^{n} \frac{n-1}{n}\left\{g_{1}\left(\hat{\boldsymbol{\delta}}_{-l}\right)-g_{1}(\hat{\boldsymbol{\delta}})\right\} .
$$

Turning to jackknife estimation of the last term, $M_{2}$, in (2.4), let $\hat{\gamma}^{B}=k\left(\boldsymbol{y}_{s}, \boldsymbol{X}_{s}, \boldsymbol{\phi}\right)$ be the Bayes predictor expressed as a function of $\boldsymbol{y}_{s}, \boldsymbol{X}_{s}$ and $\phi$ so that $\hat{\gamma}^{E B}=k\left(\boldsymbol{y}_{s}, \boldsymbol{X}_{s}, \hat{\boldsymbol{\phi}}\right)$. Now replace $\hat{\boldsymbol{\phi}}$ by $\hat{\boldsymbol{\phi}}_{-l}$ to get $\hat{\gamma}_{-l}^{E B}=k\left(y_{s}, X_{s}, \hat{\boldsymbol{\phi}}_{-l}\right)(l=1, \ldots, n)$. Then an jackknife estimator of $M_{2}$ is given by

$$
\hat{M}_{2 J}=\sum_{l=1}^{n} \frac{n-1}{n}\left(\hat{\gamma}_{-l}^{E B}-\hat{\gamma}^{E B}\right)^{2} .
$$

By taking the sum of (2.5) and (2.6), a jackknife estimator of $M S P E\left(\hat{\gamma}^{E B}\right)$ is obtained as

$$
\operatorname{mspe}_{J}\left(\hat{\gamma}^{E B}\right)=\hat{M}_{1 J}+\hat{M}_{2 J} .
$$

Next, we consider a hierarchical Bayesian framework to predict the population means $\gamma$. To this end, we begin with the following model:

I. $y_{i} \mid \alpha, \beta, \sigma_{e}^{2} \stackrel{i n d}{\sim} N\left(\alpha+\beta x_{i}, \sigma_{e}^{2}\right), i=1, \ldots, n$ where $e_{i} \stackrel{i i d}{\sim} N\left(0, \sigma_{e}^{2}\right)$.

II. $X_{i} \mid x_{i}, \sigma_{\eta}^{2} \stackrel{i n d}{\sim} N\left(x_{i}, \sigma_{\eta}^{2}\right), i=1, \ldots, n$ where $\eta_{i} \stackrel{i i d}{\sim} N\left(0, \sigma_{\eta}^{2}\right)$.

III. $x_{i} \stackrel{i i d}{\sim} N\left(\mu_{x}, \sigma_{x}^{2}\right)$.

IV. $\alpha, \beta, \mu_{x}, \sigma_{e}^{2}, \sigma_{\eta}^{2}, \sigma_{x}^{2}$ are mutually independent with $\alpha, \beta, \mu_{x} \stackrel{i i d}{\sim}$ uniform $(-\infty, \infty), \sigma_{e}^{2} \sim$ $I G\left(a_{e} / 2, b_{e} / 2\right), \sigma_{\eta}^{2} \sim I G\left(a_{\eta} / 2, b_{\eta} / 2\right), \sigma_{x}^{2} \sim I G\left(a_{x} / 2, b_{x} / 2\right)$. Here $I G(a, b)$ denotes an inverse gamma distribution with pdf $f_{a, b}(z) \propto \exp (-a / z) z^{(-b-1)} I_{[z>0]}$.

The implementation of the Bayesian procedure is greatly facilitated by the MCMC numerical integration technique, in particular the Gibbs sampler. This requires generating samples from the full conditionals of each of $x_{i}, \alpha, \beta, \mu_{x}, \sigma_{e}^{2}, \sigma_{\eta}^{2}$ and $\sigma_{x}^{2}$ given the remaining parameters and the data. The details are given below. 
By the HB model I to IV, the joint posterior distribution is given by

$$
\begin{aligned}
& \pi\left(\alpha, \beta, \mu_{x}, \sigma_{e}^{2}, \sigma_{\eta}^{2}, \sigma_{x}^{2} \mid \boldsymbol{y}_{s}, \boldsymbol{X}_{s}\right) \propto\left(\sigma_{e}^{2}\right)^{-\frac{n}{2}} \exp \left[-\frac{1}{2 \sigma_{e}^{2}} \sum_{i=1}^{n}\left(y_{i}-\alpha-\beta x_{i}\right)^{2}\right] \\
& \times\left(\sigma_{\eta}^{2}\right)^{-\frac{n}{2}} \exp \left[-\frac{1}{2 \sigma_{\eta}^{2}} \sum_{i=1}^{n}\left(X_{i}-x_{i}\right)^{2}\right] \times\left(\sigma_{x}^{2}\right)^{-\frac{n}{2}} \exp \left[-\frac{1}{2 \sigma_{x}^{2}} \sum_{i=1}^{n}\left(x_{i}-\mu_{x}\right)^{2}\right. \\
& \times \exp \left[-\frac{a_{x}}{2 \sigma_{x}^{2}}\right]\left(\sigma_{x}^{2}\right)^{-\left(b_{x} / 2+1\right)} \times \exp \left[-\frac{a_{\eta}}{2 \sigma_{\eta}^{2}}\right]\left(\sigma_{\eta}^{2}\right)^{-\left(b_{\eta} / 2+1\right)} \times \exp \left[-\frac{a_{e}}{2 \sigma_{e}^{2}}\right]\left(\sigma_{e}^{2}\right)^{-\left(b_{e} / 2+1\right)} .
\end{aligned}
$$

Then the full conditionals are obtained as follows:

(i) $\left[\alpha \mid \boldsymbol{x}, \beta, \mu_{x}, \sigma_{e}^{2}, \sigma_{\eta}^{2}, \sigma_{x}^{2}, \boldsymbol{y}, \boldsymbol{X}\right] \sim N\left(\bar{y}-\beta \bar{x}, \frac{\sigma_{e}^{2}}{n}\right)$;

(ii) $\left[\beta \mid \boldsymbol{x}, \alpha, \mu_{x}, \sigma_{e}^{2}, \sigma_{\eta}^{2}, \sigma_{x}^{2}, \boldsymbol{y}, \boldsymbol{X}\right] \sim N\left(\frac{\sum_{i=1}^{n}\left(y_{i}-\alpha\right) x_{i}}{\sum_{i=1}^{n} x_{i}^{2}}, \frac{\sigma_{e}^{2}}{\sum_{i=1}^{n} x_{i}^{2}}\right)$;

(iii) $\left[\mu_{x} \mid \boldsymbol{x}, \alpha, \beta, \sigma_{e}^{2}, \sigma_{\eta}^{2}, \sigma_{x}^{2}, \boldsymbol{y}, \boldsymbol{X}\right] \sim N\left(\bar{x}, \frac{\sigma_{e}^{2}}{n}\right)$;

(iv) $\left[\sigma_{e}^{2} \mid \boldsymbol{x}, \alpha, \beta, \mu_{x}, \sigma_{\eta}^{2}, \sigma_{x}^{2}, \boldsymbol{y}, \boldsymbol{X}\right] \sim \operatorname{IG}\left(\frac{1}{2}\left\{\sum_{i=1}^{n}\left(y_{i}-\alpha-\beta x_{i}\right)^{2}+a_{e}\right\}, \frac{n+b_{e}}{2}\right)$;

(v) $\left[\sigma_{\eta}^{2} \mid \boldsymbol{x}, \alpha, \beta, \mu_{x}, \sigma_{e}^{2}, \sigma_{x}^{2}, \boldsymbol{y}, \boldsymbol{X}\right] \sim I G\left(\frac{1}{2}\left\{\sum_{i=1}^{n}\left(X_{i}-x_{i}\right)^{2}+a_{\eta}\right\}, \frac{n+b_{\eta}}{2}\right)$;

(vi) $\left[\sigma_{x}^{2} \mid \boldsymbol{x}, \alpha, \beta, \mu_{x}, \sigma_{e}^{2}, \sigma_{\eta}^{2}, \boldsymbol{y}, \boldsymbol{X}\right] \sim I G\left(\frac{1}{2}\left\{\sum_{i=1}^{n}\left(x_{i}-\mu_{x}\right)^{2}+a_{x}\right\}, \frac{n+b_{x}}{2}\right)$;

(vii) $\left[x_{i} \mid \alpha, \beta, \mu_{x}, \sigma_{e}^{2}, \sigma_{\eta}^{2}, \sigma_{x}^{2}, \boldsymbol{y}, \boldsymbol{X}\right] \stackrel{i n d}{\sim} N\left[\left(\beta^{2} \sigma_{e}{ }^{-2}+\sigma_{\eta}^{-2}+\sigma_{x}^{-2}\right)^{-1} \times\left\{\left(y_{i}-\alpha\right) \beta \sigma_{e}^{-2}+X_{i} \sigma_{\eta}^{-2}+\right.\right.$ $\left.\left.\mu_{x} \sigma_{x}^{-2}\right\},\left(\beta^{2} \sigma_{e}^{-2}+\sigma_{\eta}^{-2}+\sigma_{x}^{-2}\right)^{-1}\right], \quad i=1, \ldots, n$.

Using Gibbs sampling, we obtain the HB estimate

$$
\hat{\gamma}^{H B}=E\left[\gamma \mid \boldsymbol{y}_{s}\right] \approx(1-f) \bar{y}_{s}+f \sum_{t=1}^{T}\left\{\mu_{y}^{(t)}+\beta^{(t)} k_{x}^{(t)}\left(\bar{X}_{\bar{s}}-\mu_{x}^{(t)}\right)\right\} / T
$$

where $\mu_{y}^{(t)}=\alpha^{(t)}+\beta^{(t)} \mu_{x}^{(t)}$ and $k_{x}^{(t)}=\sigma_{x}^{2(t)} /\left(\sigma_{x}^{2(t)}+\sigma_{\eta}^{2(t)}\right)$. And the corresponding posterior variance is given by

$$
\begin{aligned}
V\left[\gamma \mid \boldsymbol{y}_{s}\right] \approx & f^{2}\left(\sum_{t=1}^{T}\left\{\mu_{y}^{(t)}+\beta^{(t)} k_{x}^{(t)}\left(\bar{X}_{\bar{s}}-\mu_{x}^{(t)}\right)\right\}^{2} / T-\left[\sum_{t=1}^{T}\left\{\mu_{y}^{(t)}+\beta^{(t)} k_{x}^{(t)}\left(\bar{X}_{\bar{s}}-\mu_{x}^{(t)}\right)\right\} / T\right]^{2}\right) \\
& +f / N \sum_{t=1}^{T}\left\{\sigma_{e}^{2(t)}+\beta^{2(t)} \sigma_{e}^{2(t)}\left(1-k_{x}^{(t)}\right)\right\} / T .
\end{aligned}
$$

\section{Numerical studies}

This section concerns the analysis of data set to illustrate the methods obtained in preceding section. We create a finite population of total size $N=200$ under the superpopulation model with $\alpha=1, \beta=2, \mu_{x}=5, \sigma_{e}^{2}=10, \sigma_{u}^{2}=10$ and $\sigma_{x}^{2}=10$. Here $\gamma=10.35487$. Then we select samples of size 10, 25, and 50 without replacement from this population. 
We conducted similar simulation studies with several cases of the parameter values, and obtained similar results.

Using the given data set $\left(y_{i}, X_{i}\right)(i=1, \ldots, n)$, we compute the sample mean, the EB estimate and the corresponding jackknife estimate of MSPE. To obtain the HB estimators, we run a Gibbs chain of size 10,000 with a burn-in of the first 5000. After burning out the first half (to eliminate any possible instability in the initial generated samples), we use the averaging principle and take the average of the HB estimates over all the remaining sets to obtain the final HB estimate. The HB estimators of the population mean $\gamma$ are the average over the remaining 5000 Gibbs samples generated. The same method is applied to calculate the posterior standard deviation (PSD). We considered small values for $a_{e}, b_{e}, a_{u}, b_{u}, a_{x}, b_{x}$ in the inverse gamma distributions for the diffused prior information.

Table 3.1 reports the sample sizes, the true value $(\gamma)$, the sample mean $\left(\bar{y}_{s}\right)$, the EB and the $\mathrm{HB}$ estimates as well as $\operatorname{RMSE}(\mathrm{EB})$ and $\operatorname{PSD}(\mathrm{HB})$ for the samples of size 10, 25, and 50.

Table 3.1 Sample means, Bayes predictors, RMSE and PSD for the sampled data.

\begin{tabular}{ccccccc}
\hline \hline $\mathrm{n}$ & $\gamma$ & $\bar{y}_{s}$ & $\hat{\gamma}^{E B}$ & $\hat{\gamma}^{H B}$ & MSPE (EB) & PSD (HB) \\
\hline 10 & 10.35487 & 14.31396 & 13.02840 & 14.29442 & 2.810525 & 2.806568 \\
25 & 10.35487 & 12.62731 & 11.24981 & 12.49025 & 1.724124 & 1.701512 \\
50 & 10.35487 & 10.61983 & 10.43887 & 10.54388 & 0.881237 & 0.855421 \\
\hline
\end{tabular}

From Table 3.1, we may find that the EB and HB predictors are well behaved in the sense that they are closer to $\gamma$ than at least the classical estimate $\bar{y}_{s}$, but the EB predictor is slightly better than the HB predictor in the closeness to $\gamma$. Also the EB and HB predictors are comparable in the view point of the precisions MSPE and PSD.

\section{Summary and conclusion}

We have derived EB and $\mathrm{HB}$ predictors of a finite population mean under a linear regression model with covariate subject to measurement error. Our simulation results have shown that EB and HB predictors are quite comparable in the closeness to $\gamma$ as well as the precisions.

\section{References}

Basu, D. (1975). Statistical inference and likelihood. Sankhya A, 37, 1-71.

Bolfarine, H. and Cordani, L. K. (1993). Estimation of a structural linear regression model with known reliability ratio. Annals of the Institute of Statistical Mathematics, 45, 531-540.

Bolfarine, H. and Zacks, S. (1992). Prediction theory for finite populations, Springer-Verlag, New York, NY.

Bolfarine, H., Zacks, S. and Sandoval, M. (1996). On predicting the population total under regression models with measurement errors. Journal of Statistical Planning and Inference, 55, 63-76.

Cassel, C., Särndal, C. E. and Wretman, J. H. (1977). Foundations of inference in survey sampling, Wiley, New York.

Chen, S. and Lahiri, P. (2002). On mean squared prediction error estimation in small ares estimation problems. In Proceedings of the Survey Research Methods Section, American Statistical Association, 473-477.

Cochran, W. (1968). Error of measurement in statistic. Technometrics, 10, 637-666. 
Fuller, W. (1975). Regression analysis for sample surveys. Shakhya C, 37, 117-132.

Fuller, W. (1987). Measurement error models. Wiley, New York.

Jiang, J., Lahiri, P. and Wan, S. M. (2002). A unified Jackknife theory for empirical best prediction with M-estimation. Annals of Statistics, 30, 1782-1810.

Torabi, M., Datta, G. S. and Rao, J. N. K. (2009). Empirical Bayes estimation of small area means under a nested error linear regression model with measurement errors in the covariates. Scandinavian Journal of Statistics, 36, 355-368. 